\title{
Distribuição da Oviposição e Dinâmica Temporal do Aedes aegypti (Linnaeus) por Meio de Ovitrampas
}

\author{
Fred Julio Costa Monteiro ${ }^{\bowtie}$, José Carlos Tavares Carvalho \& Raimundo Nonato Picanço Souto
}

Universidade Federal do Amapá, e-mail: fredjulio@gmail.com (Autor para correspondência ${ }^{\circledR}$ ), farmacos@unifap.br, rnpsouto@unifap.br.

\section{EntomoBrasilis 7 (3): 188-192 (2014)}

\begin{abstract}
Resumo. O presente estudo teve como objetivo monitorar a dispersão do Aedes aegypti (Linnaeus) por meio de ovitrampas e correlacionar com as variáveis ambientais durante período aproximado de um ano na cidade de Macapá, AP, Brasil. Foram instaladas um total de 66 ovitrampas em 4 zonas da cidade: oeste, sul norte e central, trocando semanalmente as armadilhas. A contagem de ovos permitiu calcular o índice de positividade de ovo (IPO) e índice de densidade de ovo (IDO). Comparou-se o IPO e o IDO entre zonas, e correlacionou-se com os dados climáticos. Durante o estudo, o IPO foi de 50,44\% e o IDO 103,79. Não houve diferença do IPO entre as zonas, apenas o IDO da zona central fora maior que as demais. O IPO e o IDO estiveram correlacionados positivamente com a pluviosidade e umidade do ar mínima e, negativamente com a umidade máxima do ar e temperatura máxima e mínima. O monitoramento do A. aegypti por meio de ovitrampa direciona complementarmente a equipe de combate ao vetor, permitindo atuar de maneira mais eficaz na área de maior positividade. Nossos resultados indicam que Macapá está similarmente infestada pelo $A$. aegypti e que estes elevados índices são influenciados fortemente pelas variáveis ambientais, sendo o período mais chuvoso com os maiores índices entomológicos.
\end{abstract}

Palavra chave: armadilha de oviposição; dengue; índice de densidade de ovo; índice de positividade de ovo.

\section{Distribution of Oviposition and Temporal Dynamics of Aedes aegypti (Linnaeus) by Ovitraps}

\begin{abstract}
This study aimed to monitor the spread of Aedes aegypti (Linnaeus) using ovitraps and correlate the results with the climate data over a period of approximately one year in the city of Macapá, AP, Brazil. A total of 66 ovitraps were installed in four areas of the city (west, south, north and central), and replaced weekly. The eggs were counted to calculate the egg positivity index (EPI) and egg density index (EDI). The EPI and EDI were compared across zones and correlated with climate data. For the period of the study, the EPI was 50.44\% and the EDI was 103.79. There was no difference in EPI across different areas, and only the EDI in the central area was greater than that in the other areas. The EPI and EDI were correlated positively with rainfall and humidity minimum and negatively with maximum humidity of air and maximum and minimum temperature. The monitoring of A. aegypti using ovitraps helps the team to combat the vector, enabling them to act more effectively in areas of higher positivity. Our results indicate that Macapa is similarly infested with $A$. aegypti and these high levels are strongly influenced by environmental variables, being the rainiest period with the largest entomological indices.
\end{abstract}

Keywords: dengue; egg density index; egg positivity index; oviposition traps.

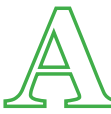

dengue é a arbovirose que se dispersa mais rapidamente no mundo (WHO 2009). Anualmente, 500 mil pessoas necessitam de hospitalização e ao menos 12,5 mil morrem em consequência da doença. Em 2013, somente nas Américas, foram relatados mais de 2,35 milhões de casos, dos quais mais de 37 mil foram da forma grave (WHO 2014).

O vírus dengue pertence ao grupo $\mathrm{B}$ dos arbovírus, família Flaviviridae, gênero Flavivirus, compreendendo 4 sorotipos: DEN 1, DEN 2, DEN 3 e DEN 4 (Rosa et al. 1997), possui como vetores mosquitos das espécies Aedes aegypti (Linnaues), principal vetor, e Aedes albopictus (Skuse), que é responsável pela transmissão da dengue na Ásia (BRASIL 2009).

No Brasil, vários fatores contribuíram para o aparecimento recorrente de epidemias de dengue, destacando-se principalmente a proliferação do vetor, o rápido crescimento demográfico associado à intensa e desordenada urbanização, a inadequada infraestrutura urbana e o aumento da produção de resíduos nãoorgânicos. Além do mais, o vetor desenvolve resistências cada vez mais evidentes às diversas formas de seu controle (AlmEIDA et al. 2009; MENDONÇA et al. 2009).

Macapá, capital do Estado do Amapá, teve o primeiro caso autóctone de dengue em 2001, quando confirmados 2.828 casos de dengue (CORREA 2007). Desde então, a doença tornou-se um grande problema de saúde pública da cidade, comportando-se de forma endêmica.

Apesar de esforços voltados a uma eminente vacina tetravalente contra dengue (LANG 2012), atualmente a prevenção ou redução da transmissão do vírus da dengue depende do controle do mosquito vetor ou interrupção do contato humano vetor (WHO 2009).

$\mathrm{Na}$ vigilância entomológica do $A$. aegypti, uma ferramenta imprescindível é a armadilha de oviposição ou ovitrampa, pois é um método sensível e econômico capaz de detectar sua presença (RAwlins et al. 1998) e que teve como pioneiros FAY \& Perry (1965). Ela pode ser usada para monitorar populações de $A$. aegypti por longos períodos (CHADEE 1990).

O presente estudo teve por objetivo monitorar a distribuição da oviposição do $A$. aegypti e sua dinâmica temporal através de ovitrampas e correlacionar com as variáveis ambientais, durante um período aproximado de um ano.

\section{MATERIAL E MÉTODOS}

Área de estudo. O estudo foi realizado no município de Macapá, capital do estado do Amapá, Brasil, o qual possui uma população de 398.204 habitantes (95,73 \% da população é urbana), de acordo com o censo demográfico de 2010, realizado 
T pelo representa 59,47\% da população do Estado (IBGE 2012).

Macapá possui o clima tropical úmido, com poucas variações de temperatura (em média de $27^{\circ} \mathrm{C}$ ), com a média anual da umidade relativa do ar de $81 \%$ e com uma pluviosidade média em torno de $2.600 \mathrm{~mm}$ (JEsus et al. 2000), sendo que o período mais seco concentra-se entre os meses de setembro a novembro (chuva trimestral abaixo de $200 \mathrm{~mm}$ ) e o mais chuvoso entre março a maio (chuva trimestral maior que $1.000 \mathrm{~mm}$ ) (SouzA \& CunHa 2010).

Inicialmente, dividiu-se a cidade em quatro zonas: oeste, sul, norte e central. De cada zona foram sorteados dois bairros para execução da pesquisa. Nestes bairros, foram instalados ovitrampas distantes $400 \mathrm{~m}$ de raio aproximadamente, localizado em nível do solo no ambiente peridomiciliar, totalizando 66 pontos amostrais, das quais 16 estavam localizados na zona oeste, 18 na zona sul, 11 na norte e 21 na central (Figura 1 ).
Azona oeste(representada pelos bairros Marabaixoe Cabralzinho) tem a média de 3,33 habitantes por domicílio. É caracterizada pela carência no fornecimento de água e de esgotamento sanitário através da rede geral. É a zona que possui menor cobertura do sistema de coleta de lixo.

A zona sul (Zerão e Fazendinha) possui a média de 4,2 habitantes por residência, com extensa área de cobertura vegetal, e é considerada a menos urbanizada.

A zona norte (Parque dos Buritis e São Lázaro) tem 6.484 residências e 26.720 habitantes, com características socioambientais semelhantes a da zona sul.

Já a zona central (Central e Jesus de Nazaré) é a mais urbanizada de todas as zonas do estudo. Reúne a maioria dos prédios públicos e a área comercial da cidade, com a média de 3,79 habitantes por residência, é a zona mais urbanizada da cidade, possui a maior cobertura da rede geral de água e esgoto, além da maioria das ruas são pavimentadas (Tabela 1).

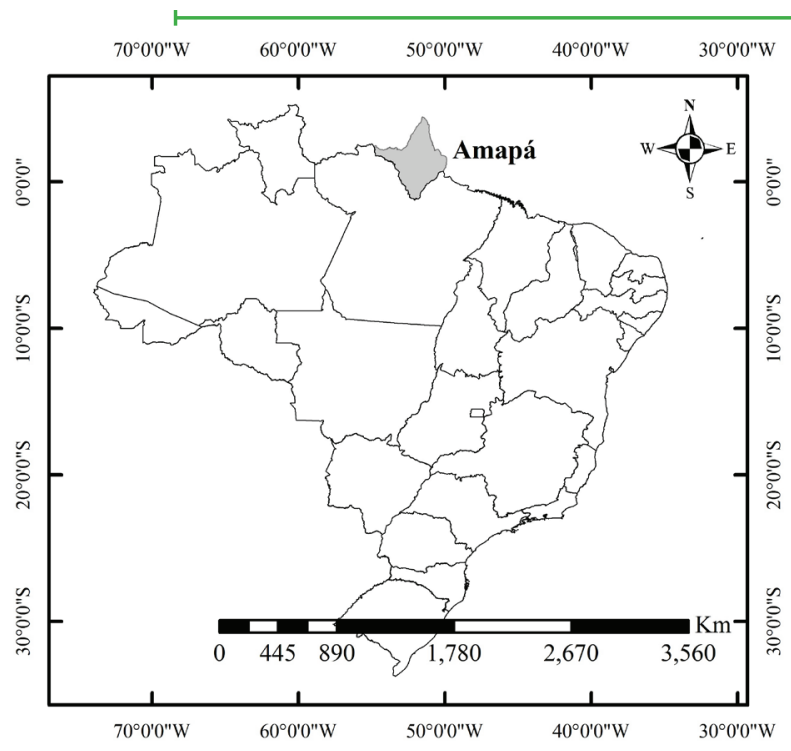

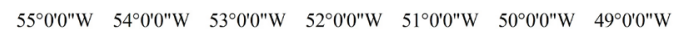
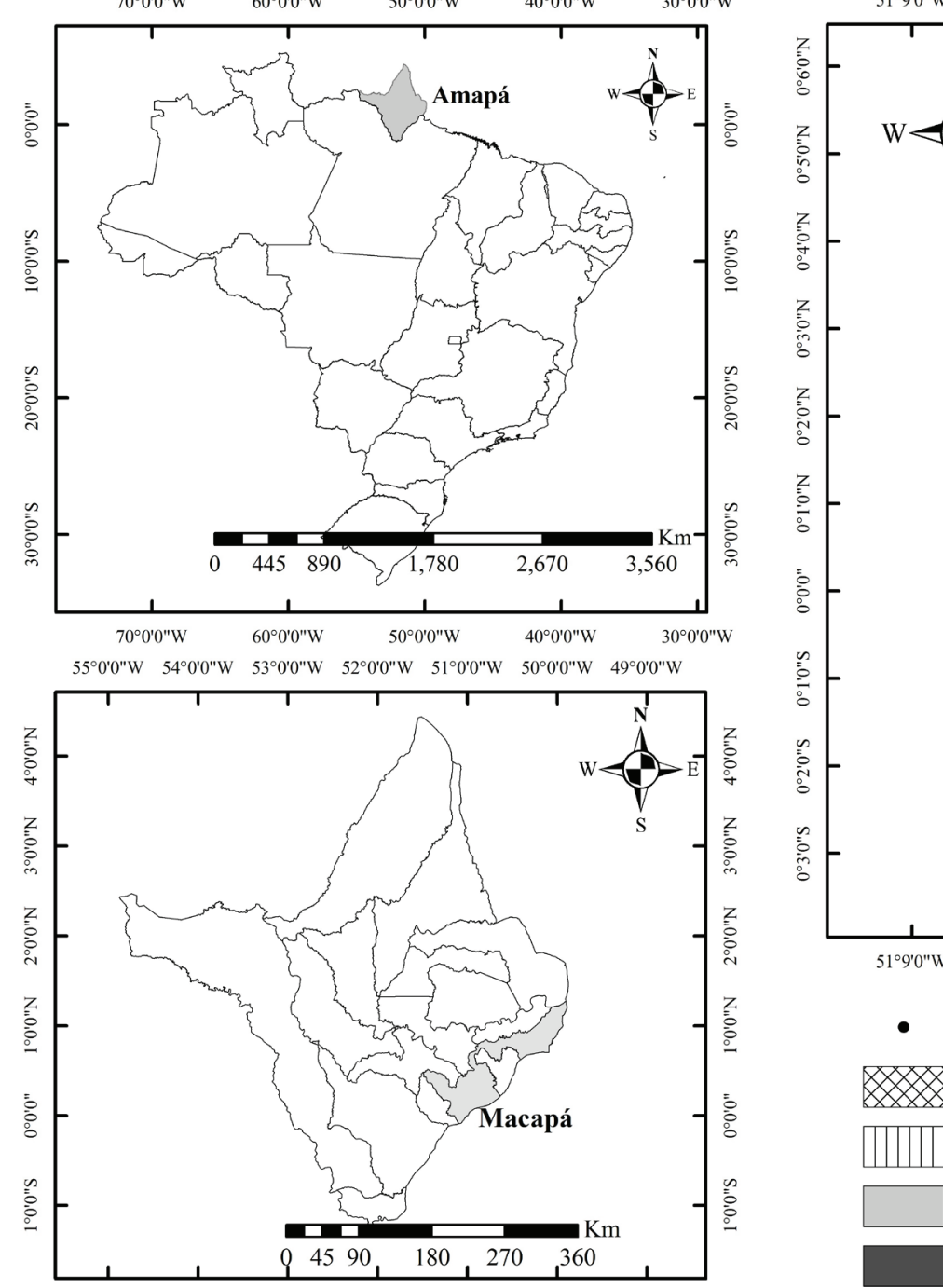

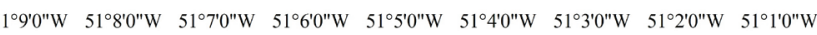
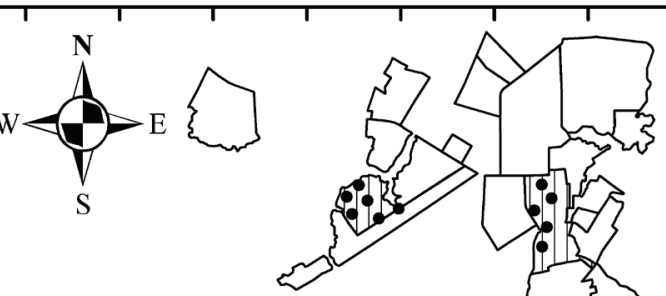

- Ovitrampa

Zona Sul

Zona Norte

Zona Central

Zona Oeste

Figura 1. Área de estudo indicando os pontos de instalação das ovitrampas.

Tabela 1. Características demográficas e sanitárias das zonas de estudo em Macapá, Amapá, Brasil, durante a semana epidemiológica 38/2011 a $35 / 2012$

\begin{tabular}{|c|c|c|c|c|}
\hline Variável & Zona Oeste & Zona Sul & Zona Norte & Zona Central \\
\hline Número de domicílios & 4.049 & 5.146 & 6.484 & 6.236 \\
\hline População residente & 13.502 & 21.609 & 26.720 & 23.659 \\
\hline \% de domicílios ligados a rede geral de distribuição de água & 7,93 & 49,73 & 27,33 & 78,3 \\
\hline \% domicílios ligados a rede geral de esgoto ou pluvial & 0,47 & 1,75 & 1,28 & 49,55 \\
\hline \% domicílio com coleta de lixo & 77,5 & 98,64 & 98,47 & 99,7 \\
\hline
\end{tabular}

Fonte: IвGE (2012). 
Ovitrampas. As ovitrampas confeccionadas foram compostas por um vaso plástico preto de $500 \mathrm{~mL}$, contendo $200 \mathrm{~mL}$ de infusão de feno a 10\% elaborada de acordo com REITER et al. (1991), tratada com diflubenzuron, contendo uma palheta de eucatex (fixada por um clipe), medindo $12 \mathrm{~cm}$ de altura e 2,5 $\mathrm{cm}$ de largura, deixando a parte áspera voltada para o centro do recipiente para favorecer a oviposição. As palhetas foram devidamente identificadas através de etiquetas, com a data e o local da instalação.

As armadilhas foram trocadas semanalmente, por um período compreendido entre a semana epidemiológica 38/2011 e a semana epidemiológica 35/2012, sendo as palhetas incineradas após contagem.

Determinação do IPO e IDO. As instalações das ovitrampas permitiram determinar semanalmente o índice de positividade de ovo (IPO), que é a porcentagem expressa pelo número de ovitrampas positivas dividida pelo número total de ovitrampas instaladas. Da mesma forma, foi calculado o índice de densidade de ovo (IDO), que é a relação entre número de ovos sobre o número de ovitrampas positivas.

Índice Rápido para o $\boldsymbol{A}$. aegypti (LIRAa). Os dados referentes aos LIRAa's foram fornecidos pelo Departamento de Vigilância em Saúde de Macapá. Por meio do LIRAa foi possível identificar os principais criadouros para o A. aegypti durante a pesquisa.

Variáveis ambientais. Os dados da pluviosidade, umidade do ar (máxima e mínima) e temperatura (máxima e mínima,) foram fornecidos pelo Núcleo de Hidrometereologia e Energias Renováveis do Instituto de Estudos e Pesquisas do Estado do Amapá, na cidade de Macapá, Amapá, Brasil (NHMET/ IEPA). A pluviosidade considerada foi a acumulada por semana epidemiológica, coincidindo assim, com o período de estudo. Similarmente, foram utilizadas as médias semanais tanto para umidade do ar (máxima e mínima) quanto para temperatura (máxima e mínima).

Análise estatística. Para realizar as análises estatísticas utilizou-se o software Bioestat 5.3 de Ayres et al. (2007).

Inicialmente foi realizada a comparação entre as quatro zonas delimitadas, pelo método estatístico de Kruskal Wallis, tanto para o IPO quanto IDO.

Também foi empregado o teste de correlação de Spearman para verificar a relação entre a pluviosidade acumulada, umidade do ar (máxima e mínima) e temperatura (máxima e mínima) com o IPO e IDO de cada zona de estudo. Para isto, os dados ambientais foram correlacionados após duas semanas com o IPO e o IDO, ou seja, as informações ambientais da semana epidemiológica $38 / 2011$, foram correlacionadas com o IPO e o IDO da semana 40/2011 e assim sucessivamente.

Ouso de métodos não paramétricos foijustificado pela distribuição não normal dos dados e a heterocedasticidade da variância, que persistiram mesmo após transformação logarítmica.

\section{RESULTADOS}

No total, foram instaladas 2.508 ovitrampas, das quais 2.339 palhetas foram coletadas, sendo que as demais 169 foram perdidas ou a ovitrampa foi esvaziada. Foram contados 122.478 ovos de $A$. aegypti em 1.180 palhetas positivas, ou seja, um IPO de 50,44\% com o IDO de 103,79.

Não houve diferença significativa do IPO entre a mediana das zonas oeste $(51,67 \%)$, sul $(55,91 \%)$, norte $(63,07 \%)$ e central (61,51\%) (Teste de Kruskall-Wallis, $\mathrm{H}=2,869 ; \mathrm{gl}=3 ; p=0,4122$ ). Porém, relacionado ao IDO, há diferença significativa entre as zonas oeste, sul, norte e central (Teste de Kruskall-Wallis, $\mathrm{H}=$ o,103; $\mathrm{gl}=3 ; p=0,0177)$, onde a mediana da zona sul $(68,5)$ foi significantemente menor do que a da zona central $(89,5)$ (Método de Dunn $p<0,05)$, não diferenciando as demais.

O IPO foi correlacionado positivamente com a pluviosidade e a umidade mínima em todas as zonas de estudo (Tabela 2). Já o IDO foi correlacionado significantemente positivo com estas variáveis nas zonas oeste, sul e norte, mas na central, não houve correlação significativa (Tabela 3 ).

Por outro lado, o IPO foi correlacionado negativamente com a umidade máxima e a temperatura máxima e mínima em todas as zonas de estudo (Tabela 2). O IDO foi correlacionado negativamente com a umidade máxima nas zonas oeste, sul e norte. Contudo, na zona central não houve correlação significativa entre o IDO no peridomicílio com a umidade máxima (Tabela $3)$.

Tabela 2. Correlação entre o IPO e as variáveis ambientais por zona de estudo durante a semana epidemiológica 38/2011 a 35/2012, em Macapá, Amapá, Brasil

\begin{tabular}{|c|c|c|c|c|c|c|c|c|c|c|c|c|}
\hline \multirow{2}{*}{$\begin{array}{l}\text { Variáveis } \\
\text { ambientais }\end{array}$} & \multicolumn{3}{|c|}{ Zona Oeste } & \multicolumn{3}{|c|}{ Zona Sul } & \multicolumn{3}{|c|}{ Zona Norte } & \multicolumn{3}{|c|}{ Zona Central } \\
\hline & $\mathbf{R S}$ & $\mathbf{T}$ & $\boldsymbol{p}_{\text {valor }}$ & RS & $\mathbf{T}$ & $\boldsymbol{p}_{\text {valor }}$ & $\mathbf{R S}$ & $\mathbf{T}$ & $\boldsymbol{p}_{\text {valor }}$ & RS & $\mathbf{T}$ & $\boldsymbol{p}_{\text {valor }}$ \\
\hline Pluviosidade & 0,613 & 4,654 & $<0,0001$ & 0,654 & 5,188 & $<0,0001$ & 0,516 & 3,617 & 0,0009 & 0,696 & 5,822 & $<0,0001$ \\
\hline Umidade máxima & $-0,568$ & $-4,142$ & 0,0002 & $-0,551$ & $-3,964$ & 0,0003 & $-0,661$ & $-5,387$ & $<0,0001$ & $-0,618$ & $-4,721$ & $<0,0001$ \\
\hline Umidade mínima & 0,645 & 5,069 & $<0,0001$ & 0,563 & 4,094 & 0,0002 & 0,626 & 4,817 & $<0,0001$ & 0,787 & 7,660 & $<0,0001$ \\
\hline $\begin{array}{l}\text { Temperatura } \\
\text { máxima }\end{array}$ & $-0,587$ & $-4,350$ & 0,0001 & $-0,588$ & $-4,332$ & 0,0001 & $-0,631$ & $-4,880$ & $<0,0001$ & $-0,805$ & $-8,144$ & $<0,0001$ \\
\hline $\begin{array}{l}\text { Temperatura } \\
\text { mínima }\end{array}$ & $-0,572$ & $-4,185$ & 0,0002 & $-0,544$ & $-3,893$ & 0,0004 & $-0,627$ & $-4,830$ & $<0,0001$ & $-0,772$ & $-7,307$ & $<0,0001$ \\
\hline
\end{tabular}

Tabela 3. Correlação entre o IDO e as variáveis ambientais por zona de estudo durante a semana epidemiológica 38/2011 a 35/2012, em Macapá, Amapá, Brasil

\begin{tabular}{|c|c|c|c|c|c|c|c|c|c|c|c|c|}
\hline \multirow{2}{*}{$\begin{array}{c}\text { Variáveis } \\
\text { ambientais }\end{array}$} & \multicolumn{3}{|c|}{ Zona Oeste } & \multicolumn{3}{|c|}{ Zona Sul } & \multicolumn{3}{|c|}{ Zona Norte } & \multicolumn{3}{|c|}{ Zona Central } \\
\hline & RS & $\mathbf{T}$ & $p_{\text {valor }}$ & RS & $\mathbf{T}$ & $p_{\text {valor }}$ & RS & $\mathbf{T}$ & $p_{\text {valor }}$ & RS & $\mathbf{T}$ & $p_{\text {valor }}$ \\
\hline Pluviosidade & 0,560 & 4,055 & 0,0003 & 0,431 & 2,867 & 0,0068 & 0,410 & 2,702 & 0,0104 & 0,306 & 1,932 & 0,0611 \\
\hline Umidade máxima & $-0,599$ & $-4,494$ & $<0,0001$ & $-0,620$ & $-4,752$ & $<0,0001$ & $-0,730$ & $-6,410$ & $<0,0001$ & $-0,209$ & $-1,286$ & 0,2065 \\
\hline Umidade mínima & 0,616 & 4,696 & $<0,0001$ & 0,441 & 2,952 & 0,0055 & 0,471 & 3,211 & 0,0028 & 0,310 & 1,961 & 0,0575 \\
\hline $\begin{array}{l}\text { Temperatura } \\
\text { máxima }\end{array}$ & $-0,680$ & $-5,568$ & $<0,0001$ & $-0,475$ & $-3,243$ & 0,0025 & $-0,509$ & $-3,553$ & 0,0011 & $-0,198$ & $-1,212$ & 0,2333 \\
\hline $\begin{array}{l}\text { Temperatura } \\
\text { mínima }\end{array}$ & $-0,657$ & $-5,238$ & $<0,0001$ & $-0,442$ & $-2,961$ & 0,0054 & $-0,527$ & $-3,721$ & 0,0007 & $-0,221$ & $-1,365$ & 0,1806 \\
\hline
\end{tabular}


Durante a pesquisa foram realizados cinco LIRAa's, sendo que nos

risco para o demais. Em todos os LIRAa's o lixo foi o principal dois primeiros Macapá foi considerada de baixo risco e de médio

criadouro encontrado (depósito do tipo D2) (Tabela 4).

Tabela 4. Resultado dos LIRAa's realizados em Macapá, durante semana epidemiológica 38/2011 a 35/2012, em Macapá, Amapá, Brasil.

\begin{tabular}{|c|c|c|c|c|c|c|c|c|c|}
\hline \multirow{2}{*}{ Semana Epidemiológica } & \multirow{2}{*}{ IIP } & \multirow{2}{*}{ IB } & \multicolumn{7}{|c|}{ ITR } \\
\hline & & & A1 & A2 & B & C & D1 & D2 & $\mathbf{E}$ \\
\hline $46 / 2011$ & 0,8 & 1,1 & 6,8 & 9,6 & 4,1 & 8,2 & 19,2 & 50,7 & 1,4 \\
\hline 02/2012 & 0,7 & 0,8 & 15,1 & 11,3 & 1,9 & 17,0 & 7,5 & 45,3 & 1,9 \\
\hline $10 / 2012$ & 2,1 & 2,5 & 6,6 & 13,2 & 3,0 & 7,8 & 9,0 & 59,2 & 1,2 \\
\hline 19/2012 & 1,8 & 2,1 & 1,4 & 13,7 & 7,9 & 7,9 & 22,3 & 44,6 & 2,2 \\
\hline $27 / 2012$ & 1,7 & 1,9 & 3,1 & 7,8 & 7,0 & 3,1 & 20,3 & 58,7 & o \\
\hline
\end{tabular}

Onde: IIP - Índice de Infestação Predial; IB: Índice de Breteau; ITR: Índice por Tipo de Recepiente; A1: Depósitos de água elevados; A2: Depósitos de armazenamento de água ao nível de solo; B: Depósitos móveis; C: Depósitos fixos; D1: Depósitos passíveis de remoção - pneus e materiais rodantes; D2: Depósitos passíveis de remoção - lixo; E: Depósitos naturais.

\section{DISCUSSÃO}

A ovitrampa mostrou-se uma ferramenta sensível à detecção da presença de fêmeas de $A$. aegypti, registrando elevado IPO quando exposta por uma semana, índice inferiores aos resultados encontrados por LOURENÇO-DE-OliveIRA (2008) no município do Rio de Janeiro (RJ) e superiores aos de SANTOS-NunEs et al. (2011), no município de Santana (AP), localizado a 18 km de Macapá.

O IPO é importante para avaliar qualitativamente a presença de A. aegypti. Neste estudo, por não apresentar diferença significativa no IPO, os resultados sugerem que os níveis de infestação são semelhantes nas diferentes áreas da cidade, mas diferenciando quanto ao IDO, que define a zona central com uma maior densidade de ovos, cujo qual se manteve elevado ao longo do estudo.

Esta importância é devida a zona central além de possuir o centro comercial de Macapá, é uma grande produtora de lixo, o qual mostrou ser o principal criadouro do vetor. Além do que, a zona central tem vários prédios públicos e muitas edificações que não possuem trabalho efetivo de eliminação e tratamento de criadouros do mosquito, conforme preconiza o PNCD (BRASIL 2002). Ademais, as equipes do Programa Municipal do Controle da Dengue, não possuem equipamentos adequados para inspeção de edifícios (recipientes do tipo A1), o que impossibilita o devido tratamento de locais de difícil acesso, ou seja, existe a perpetuação de criadouros permanentes capazes de manter uma elevada positividade e densidade das ovitrampas.

As variáveis climáticas interferem diretamente na presença do $A$. aegypti. Para Hemme et al. (2010), a interação entre temperatura, umidade relativa e pluviosidade, impactam na sobrevivência e disponibilidade de adultos e locais de oviposição. Segundo Costa et al. (2010) a elevação da temperatura e a redução da umidade durante o período mais seco, pode influenciar negativamente sobre a biologia do vetor, além de também influenciar a positividade e densidade das ovitrampas (Costa et al. 2008).

Nestapesquisa, as elevadastemperaturas tiveram efeitos negativos sobre o IDO e IPO, pois de acordo com BESERRA et al. (2006) temperaturas acima de $34^{\circ} \mathrm{C}$ prejudicam o desenvolvimento do A. aegypti, fato este observado neste estudo quando no período compreendido entre a semana epidemiológica 38/2011 e 3/2012 a temperatura máxima ultrapassou este limite.

O aumento da pluviosidade disponibiliza maior oferta de criadouros e consequentemente, elevando a densidade vetorial e a susceptibilidade da ovitrampa de ser positivada. Assim, a pluviosidade é uma importante variável climática no monitoramento por meio de ovitrampa, sendo que neste estudo, correlacionando-se positivamente com o IPO e IDO (exceto na zona central), resultados estes diferentes dos encontrados por KonAN et al. (2013), que verificaram associação apenas entre o
IPO e a pluviosidade, mas não entre o IDO. Da mesma forma ZEIDLER et al. (2008) não acharam correlação entre o IDO e a pluviosidade.

Outro fator que pode contribuir para não relação entre a pluviosidade com o IPO e IDO é quando estes índices entomológicos são elevados, mantido por condições ambientais favoráveis, principalmente a presença de criadouros permanentes, a pluviosidade pode não influenciar na presença e densidade do vetor.

Na presente pesquisa, a correlação do IPO e IDO com a pluviosidade foi significativa após duas semanas, pois este é o período necessário para que as formas imaturas se desenvolvam até a fase adulta e assim haja o voo nupcial, por conseguinte repasto sanguíneo e posterior oviposição, positivando as armadilhas.

Em 2010, Macapá possuía apenas 42,6\% e 5,6\% dos domicílios atendidos rede de água e esgoto, respectivamente (SNSA 2012), além do que a coleta de lixo na cidade é irregular. Este conjunto de fatores favorece para proliferação do mosquito, uma vez que contribui para o uso de recipientes de armazenamento de água, fossas, além do acúmulo de lixo em via pública (Tauil 2001). Esta falta de infraestrutura reflete na elevação da disponibilidade de criadouro para o $A$. aegypti, que de acordo com os resultados dos LIRAa's realizados durante a pesquisa, indicam que os depósitos passíveis de remoção (principalmente o lixo) e os de armazenamento de água como principais criadouros para o $A$. aegypti em Macapá.

A estratificação do IPO para orientar o trabalho das equipes de campo é uma estratégia que deve ser utilizada na rotina dos programas de controle da dengue e pode ser desenvolvida semanalmente.

O monitoramento do A. aegypti por meio de ovitrampa direciona complementarmente a equipe de campo de combate ao vetor, permitindo atuar de maneira mais eficaz na área de abrangência da armadilha, concentrando esforços nas áreas de IPO mais elevado.

Por fim, nossos resultados indicam que Macapá está similarmente infestada pelo $A$. aegypti e que estes elevados índices são influenciados fortemente pelas variáveis ambientais, sendo o período mais chuvoso com os maiores índices entomológicos.

\section{AGRADECIMENTOS}

A equipe de entomologia do Departamento de Vigilância em Saúde de Macapá, pelo apoio na realização da pesquisa e pelo fornecimento dos dados do LIRAa. Ao NHMET/IEPA pelo fornecimento dos dados ambientais. 


\section{REFERÊNCIAS}

Almeida, A.S.D., R.D.A. Medronho \& L.I.O. Valência, 2009. Análise espacial da dengue e o contexto socioeconômico no município do Rio de Janeiro, RJ. Revista de Saúde Pública, 43: 666-673.

Ayres, M., M. Ayres Jr, D.L. Ayres \& A.A.S. Santos, 2007. BioEstat 5.3 - Statistical applications in the areas of biomedical sciences. Disponível em: <http://www.mamiraua.org.br $>$.

Beserra, E.B., F.P. Castro, J.W. Santos, T.D.A. Santos \& C.R. Fernandes, 2006. Biology and thermal exigency of $s$ (L.) (Diptera: Culicidae) from four bioclimatic localities of Paraiba. Neotropical Entomology, 35: 853-860.

Brasil, 2002. Programa nacional de controle da dengue. Brasília, Ministério da Saúde, 32p.

Brasil, 2009. Diretrizes nacionais para a prevenção e controle de epidemias de dengue. Brasília, Ministério da Saúde, 16op.

Chadee, D.D., 1990. Aedes aegypti surveillance in Tobago, West Indies (1983-88), 1990. Journal of the American Mosquito Control Association, 6: 140-150.

Correa, A.P.S.A., 2007. Distribuição espacial de criadouros de Aedes (Stegomya) aegypti Linneus 1762 (Diptera: Culicidae) em pontos georreferenciados de dois bairros da cidade de Macapá-Ap: área endêmica de dengue. Dissertação (Mestrado em Biologia de Agentes Infecciosos e Parasitários) - Universidade Federal do Pará. 101p.

Costa, E.P.A.A., E.M.M.S. Santos, J.C. Correia \& C.M.R. Albuquerque, 2010. Impact of small variations in temperature and humidity on the reproductive activity and survival of Aedes aegypti (Diptera, Culicidae). Revista Brasileira de Entomologia, 54: 488-493.

Costa, F.S., J.J. Silva, C.M. Souza \& J. Mendes, 2008. Population dynamics of Aedes aegypti (L) in an urban area with high incidence of dengue. Revista da Sociedade Brasileira de Medicina Tropical, 41: 309-312.

Fay, R.W. \& A.S. Perry, 1965. Laboratory studies of ovipositional preferences of Aedes aegypti. Mosquito News, 25: 276-281.

Hemme, R.R., C.L. Thomas, D.D. Chadee \& D.W. Severson, 2010. Influence of urban landscapes on population dynamics in a short-distance migrant mosquito: evidence for the dengue vector Aedes aegypti. PLoS Neglected Tropical Diseases, 4: 1-9.

IBGE, 2012. Sinopse do Censo de 2010. IBGE, 1: 1. Disponível em: $<$ http://www.ibge.gov.br/estadosat/perfil.php?sigla=ap $>$. [Acesso em: 01.07.2012].

Jesus, E.S., P.H.L. Gonçalves \& M.C.F. Oliveira, 2000. The variability of meteorological elements associated with the El Niño phenomenon in the city of Macapá. XI Congresso Brasileiro de Meteorologia, 1: 1009-1013.

Konan, Y.L., Z.I. Coulibaly, A.B. Kone, K.D. Ekra, J.M. Doannio, M. Dosso \& P. Odehouri-Koudou, 2013. Species composition and population dynamics of Aedes mosquitoes, potential vectors of arboviruses, at the container terminal of the autonomous port of Abidjan, Cote d'Ivoire. Parasite, 20: 1-6.

Lang, J., 2012. Development of Sanofi Pasteur tetravalent dengue vaccine. Revista do Instituto de Medicina Tropical de São Paulo, 18: 15-17.

Lourenço-de-Oliveira, R., J.B. Lima, R. Peres, F.C. Alves, A.E. Eiras \& C.T. Codeco, 2008. Comparison of different uses of adult traps and ovitraps for assessing dengue vector infestation in endemic areas. Journal of the American Mosquito Control Association, 24: 387-392.

Mendonça, F.A., A.V.E. Souza \& D.D.A. Dutra, 2009. Public health, urbanization and dengue's fever in Brazil. Sociedade \& Natureza, 21: 257-269.

Rawlins, S.C., R. Martinez, S. Wiltshire \& G. Legall, 1998. A comparison of surveillance system for the dengue vector Aedes aegypti in Porto of Spain, Trinidad. Journal of the American Mosquito Control Association, 14: 131-136.

Reiter, P., M.A. Amador \& N. Colon, 1991. Enhancement of the CDC ovitrap with hay infusions for daily monitoring of Aedes aegypti populations. Journal of the American Mosquito Control Association, 7: 52-55.

Rosa, A.P.R.T., P.F.C. Vasconcelos, P.F. Pinheiro, J.F.S.T. Rosa \& S.G. Rodrigues, 1997. Dengue, p. 227. In: Leão, R.N.Q. Doenças Infecciosas e Parasitárias: enfoque amazônico. 1, Belém, Cejup, 885p.

Santos-Nunes, L.D., R.B.R. Trindade \& R.N.P. Souto, 2011. Evaluation of attractiveness of ovitraps to Aedes (Stegomyia) aegypti Linneus (Diptera: Culicidae) in Hospitality district, Santana, Amapá. Biota Amazônia, 1: 26-31.

SNSA, 2012. Sistema Nacional de Informações sobre Saneamento: diagnóstico dos Serviços de Água e Esgotos - 2010. 1, Brasília: Ministério das Cidades. 448p.

Souza, E.B. \& A.C. Cunha, 2010. Precipitation climatology in Amapá and Large-scale Climate Mechanisms, p 177. In: Cunha, A., E.B. Souza \& H.F.A. Cunha. Weather, climate and water resources: results of the REMETAP project in the state of Amapá. 1, Macapá, IEPA, 216p.

Tauil, P.L., 2001. Urbanização e ecologia do dengue. Cadernos de Saúde Pública, 17: 99-102.

WHO (Word Health Organization), 2009. Dengue: guidelines for diagnosis, treatment, prevention and control. 1, TDR, 147p.

WHO (Word Health Organization), 2014. Dengue and severe dengue. Ficha técnica 117. Disponível em: <http://www. who.int/mediacentre/factsheets/fs117/en/>. [Acesso em: 09.08.2014].

Zeidler, J.D., P.O.A. Acosta, P.P. Barreto \& J.S. Cordeiro, 2008. Vírus dengue em larvas de Aedes aegypti e sua dinâmica de infestação, Roraima, Brasil. Revista de Saúde Pública, 42: 986-991.

\section{Recebido em: 15/o1/2014}

Aceito em: 17/09/2014

\section{Como citar este artigo:}

Monteiro, F.J.C., J.C.T. Carvalho \& R.N.P. Souto, 2014. Distribuição da Oviposição e Dinâmica Temporal do Aedes aegypti (Linnaeus) por Meio de Ovitrampas. EntomoBrasilis, 7 (3): 188-192.

Acessível em: doi:10.12741/ebrasilis.v7i3.419
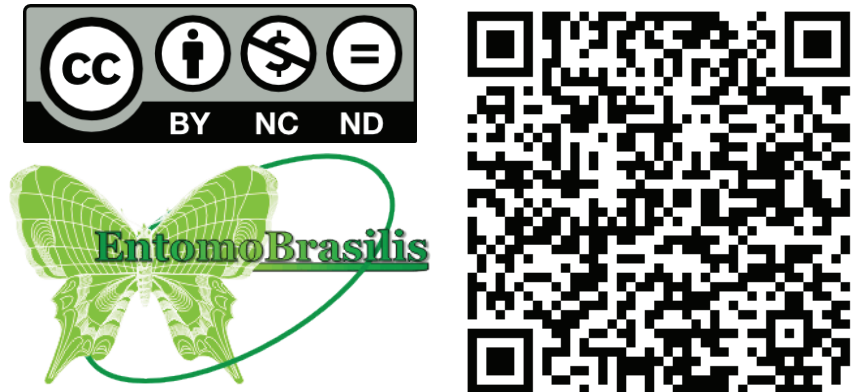